\title{
Aplicación de análisis discriminante para evaluar la productividad como resultado de la certificación BASC en las empresas de la ciudad de Cartagena
}

Tomás José Fontalvo

Herrera

Facultad de Ciencias Económicas, Universidad de Cartagena tfontalvoh@unicartagena.edu.co

\section{Resumen}

En este artículo de investigación se presenta la evaluación de la productividad en las empresas de la ciudad de Cartagena como resultado de la certificación de éstas en la Coalición Empresarial Anticontrabando (CEAC, por sus siglas en inglés). En la metodología utilizada se calcularon los indicadores de productividad a las 23 empresas certificadas en CEAC. En seguida, se utilizó la técnica de análisis discriminante para explicar la pertenencia y discriminación de cada grupo de los indicadores de productividad evaluados; como resultado de ello se obtuvo la correlación existente entre las empresas certificadas y el incremento en los índices de productividad de los años 2008 y 2010. De la función discriminante obtenida y los estadísticos analizados se puede concluir que la certificación en la norma Business Anti-Smuglin Coalition (BASC, por sus siglas en inglés) no mostró un mejoramiento en los indicadores seleccionados, pero presentó diferencias significativas en los indicadores Utilidad bruta/Valor agregado (IP1), Utilidad operacional/ Capital operativo (IP5) y Utilidad neta/Capital operativo (IP6).

Palabras clave: productividad, certificación, indicador, análisis discriminante. 


\title{
Application of discriminant analysis to assess productivity as a result from the BASC certification in Cartagena companies
}

\begin{abstract}
This research paper presents the evaluation of productivity in companies in the city of Cartagena as a result of the certification of said companies in the Business Anti-Smuggling Coalition (BASC). The methodology used calculated productivity indicators at 23 companies certified at BASC. Next, we used the Discriminant Analysis technique to explain the belonging and discrimination of each group of productivity indicators evaluated, resulting in the correlation between certified companies and increased productivity indices for 2008 and 2010. From the discriminant function obtained and the statistics analyzed, it can be concluded that certification in BASC standard showed no improvement in the indicators selected, but presented significant differences in the Gross Profit / Value Added (IP1), Operating Income / Operating Capital (IP5) and Net Income / Operating Capital (IP6) indicators.
\end{abstract}

Keywords: productivity, certification, indicator, discriminant analysis.

\section{Introducción}

Dada la importancia de la productividad para el incremento de la eficiencia y eficacia en las organizaciones, en este artículo se muestra la evaluación de la productividad como resultado de la certificación Coalición Empresarial Anticontrabando (Business Anti-Smugling Coalition, BASC, por sus siglas en inglés) en las empresas de Cartagena de Indias, Colombia, y la incidencia de ésta en la productividad.

Asimismo, se presentan los retos del comercio internacional, la importancia de un comercio seguro, los beneficios de la seguridad en el comercio, los sistemas de gestión del Business Anti-Smugling Coalition (BASC) para la seguridad en el comercio, los indicadores de productividad y la evaluación del impacto sobre la productividad mediante el análisis discriminante en las empresas de la ciudad de Cartagena.

Para evaluar el impacto de la productividad como resultado de la certificación BASC en las empresas de la ciudad de Cartagena, se desarrolló una metodología para el análisis de los datos de aquellas empresas certificadas en BASC que pre- 
sentaron sus estados financieros en la Superintendencia de Sociedades en los años 2008 y 2010; para ello se utilizó la técnica de análisis discriminante (ADM).

Finalmente, se presentan los resultados de la evaluación de la productividad financiera como resultado de la certificación BASC y la verificación de supuestos a partir de las pruebas Saphiro \& Wilk y de Box para comprobar la normalidad e igualdad de matrices varianza-covarianza, respectivamente, de las variables objeto de estudio que permitieron analizar diferencias significativas y evolución en los indicadores seleccionados.

\section{Marco teórico}

\section{Los retos del comercio internacional}

Las características actuales del mercado y la tendencia de los países hacia la apertura económica de sus fronteras implican una mayor complejidad en los procesos y operaciones de las empresas. La conquista de nuevos mercados trae consigo una reconfiguración de la cadena de suministros que día a día se va conformando como una red única de tránsito obligatorio para los productos y servicios implicados en cualquier transacción comercial que se lleve a cabo. A medida que se avanza en la apertura de los mercados también se hacen más rigurosas las medidas para garantizar un comercio seguro; esto es, la aplicación de procedimientos que garanticen que resulten productos - desde su elaboración y transporte, sobre todo en este último - de alta calidad y con los niveles de confianza con el fin de satisfacer las necesidades del cliente y, al mismo tiempo, preservar la seguridad no sólo de ellos, sino también de las organizaciones, sus empleados e incluso de los mismos estados.

Debido a lo anterior se han diseñado e implementado sistemas de gestión cuyo objetivo es proveer a las empresas de herramientas para blindar sus procesos y productos de cualquier factor o fenómeno que pueda generar no conformidades o afectar la integridad de los productos; así lo señala Ibarra (2008) quien sustenta que las empresas deben concentrar sus esfuerzos en la estandarización de sus procesos, mientras que los gobiernos en la coordinación de sus instituciones para alcanzar los logros anteriormente señalados. En el ámbito empresarial las organizaciones se preocupan más por su participación en el mercado; en este sentido, todas sus actividades están orientadas a lograr la efectividad en su cadena de abas- 
tecimiento y distribución mediante estrategias que le permitan disminuir costos y aumentar la calidad de sus productos y conseguir el aumento de su productividad y competitividad (Fariñas, 2011).

Ibarra (2007) concluye que a medida que se expanden las actividades humanas también se incrementan los riesgos en materia ambiental, sanitaria, financiera, entre otras, que generan dichas actividades; además, agrega que la responsabilidad fundamental recae sobre todo en los empresarios y en los gobiernos quienes deben buscar la forma de minimizar los impactos negativos y evadir las amenazas que representan las transacciones comerciales a gran escala. Entre los países que exportan e importan deben establecerse acuerdos colaborativos y estratégicos con el fin de que los productos que estén ingresando al país no afecten negativamente la normalidad de las actividades económicas, lo cual se lograría mediante un adecuado control de las operaciones logísticas y de los procesos que componen la cadena de abastecimiento.

Debido a la actual complejidad para la distribución de los productos o servicios, han ingresado gran cantidad de actores y creado nuevos niveles de intermediación, lo que aumenta el riesgo potencial de los procesos y los productos al interior de la cadena de suministro (Pfohl et al., 2010; Manuj y Mentzer, 2008).

\section{La importancia de un comercio seguro}

Por lo anterior, han surgido estándares que buscan generar un comercio seguro. Generalmente se asocia la palabra comercio con las actividades relacionadas con la compra y venta de productos y servicios; desde esa perspectiva, se puede entender que el comercio seguro se limita única y exclusivamente a la seguridad en las transacciones, pero es un enfoque muy reducido porque además se debe asegurar que los productos vinculados a dichas transacciones cumplan con los requerimientos de seguridad establecidos desde el mismo momento en que se empiezan a elaborar, incluyendo las materias primas y los componentes utilizados en su fabricación. Por ello, la seguridad de la compra y venta de los productos dependerá de las medidas de control establecidas a lo largo de toda la red que representa la cadena de suministro y todas las actividades sobre las que ésta se soporta.

Tal como se mencionó, para lograr un comercio completamente seguro se vinculan todos los componentes de la cadena. Al respecto, Sarathy (2006) sustenta que los esfuerzos en seguridad requieren de una relación en la que se colabore con todos 
los socios - a través de la cadena de suministro - e incluso con los gobiernos; igualmente plantea que los costos incurridos en el corto plazo por adoptar medidas de seguridad pueden ser equilibrados a largo plazo con el mejoramiento en el desempeño de la cadena de suministro y el mejoramiento de las relaciones con los clientes.

También identifica algunos factores que son objeto de medidas de seguridad dentro de un modelo generalizado de la cadena de suministro; entre ellos destacan los objetivos estratégicos, las actividades, los procesos, las instalaciones de producción, las instalaciones y medios de transporte, la distribución física de los productos, el flujo de información, los recursos humanos y relaciones, las reglamentaciones gubernamentales.

De acuerdo con lo anterior, los sistemas de gestión en control y seguridad impactan sobre algunos factores claves en las organizaciones y afectan de algún modo su productividad y rentabilidad, ya que esta última no es más que un reflejo de la efectividad y desempeño organizacional (Chacón, 2007; Valdevira et al., 2009).

Por otra parte, Díaz (2008) señala que la aplicación de medidas de seguridad requiere del despliegue de una serie de estrategias para poder realizar transacciones en el nivel internacional, las cuales están relacionadas con las medidas tomadas por las empresas para asegurar y proteger tanto los productos como la cadena logística; ello exige cambios al interior de las estructuras de las empresas. En este sentido, Samiee (2007) destaca que la coordinación y las alianzas para la administración de la cadena de suministro son unas de las estrategias más efectivas para enfrentar la competitividad de los mercados actuales.

Según Maruchek et al. (2011), son cuatro los factores los que se deben estudiar profundamente para lograr total seguridad en las transacciones comerciales: colaboración con los gobiernos en el desarrollo de nuevas herramientas de regulación que incentiven la seguridad; mejores metodologías para la gestión de la información de los productos durante todo su ciclo de vida desde su diseño hasta su desecho; tecnologías para el seguimiento a lo largo de la cadena de suministro; y la construcción de relaciones amigables con los proveedores y los clientes. 


\section{Beneficios de la seguridad en el comercio}

Los sistemas de gestión en control y seguridad se han convertido en una de las alternativas para disminuir los riesgos que implica poseer procesos complejos e integrar la cadena de suministros. Son muchos los riesgos a los que se enfrentan las empresas que pueden afectar la integridad de los productos de los servicios y hasta de la misma organización (Young y Esqueda, 2005).

Asimismo, las empresas, de manera interna, pueden obtener grandes beneficios, algunos de los cuales se traducen en un incremento de la productividad y en mejores resultados financieros. Blanchard (2006) menciona un estudio realizado por la Universidad de Stanford en el cual se dan a conocer algunos de los beneficios que presentan las empresas que han implementado sistemas de gestión para la seguridad en la cadena de suministros; entre ellos se encuentran los siguientes: mejoramiento en la seguridad del producto; mejoramiento en la gestión de inventario; mejoramiento en la visibilidad de la cadena de suministro; mejoramiento en la manipulación del producto; aumento en la rapidez de los procesos; agilidad en la solución de problemas; aumento en la satisfacción del cliente.

Por su parte, Correa y Gómez (2010) concluyen que los sistemas de gestión en control y seguridad buscan establecer y evaluar las amenazas que enfrentan las empresas con el fin de garantizar la seguridad en su cadena de suministro y en sus transacciones nacionales e internacionales; asimismo, aseguran que el cumplimiento de la reglamentación que establece estándares de seguridad y la atención adecuada de la demanda de los clientes produce eficiencia en las operaciones de comercio y distribución, lo cual genera ventajas competitivas para las empresas y los países.

Lo señalado previamente hace necesario el establecimiento de estructuras que faciliten el procesamiento de la información y el análisis de todas las actividades para la toma de decisiones, la planificación y el control de las operaciones en el comercio internacional (Pinzón et al., 2011).

Sistema de gestión BASC para la seguridad en el comercio

La Coalición Empresarial Anticontrabando o Business Anti-Smuggling Coalition (BASC, por sus siglas en inglés) nace como una respuesta alternativa para responder a todas aquellas amenazas a las que el comercio de productos o servicios se 
ve enfrentado a diario. Nace en 1996 como una propuesta de la empresa Mattel con el fin de implementar actividades y procedimientos para que las empresas que desarrollan operaciones de comercio internacional no fueran objetos de acciones ilícitas de organizaciones delictivas para el transporte de armas o drogas, así como para frenar la gran cantidad de robos y de cargamentos en mal estado que se estaban presentando. El éxito de la propuesta fue tan alto que se tuvo la necesidad de crear una organización en el nivel internacional que certificara la estandarización de dichos procesos (BASC Colombia, 2010).

El sistema de gestión BASC utiliza un enfoque basado en procesos, lo que representa para la empresa muchas utilidades y beneficios; por ejemplo, la integración de los procesos para el alcance de los logros, confianza a las partes interesadas sobre resultados consistentes, transparencia en las operaciones, reducción de los costos y del tiempo a través del uso adecuado de los recursos y la mejora de los resultados. Según Osorio (2010), las empresas que obtienen la certificación BASC se benefician porque ésta brinda mayor seguridad en los procesos, lo que les permite aumentar el número de transacciones comerciales que realizan a través de la captación de nuevos clientes.

Tal como se mencionó, las organizaciones que no ofrezcan garantías de seguridad pueden ser excluidas de cualquier proceso comercial. Una de las ventajas que presenta BASC es la posibilidad de que la empresa se convierta en un operador económico autorizado, denominación que se otorga a empresas que están involucradas en la compra y venta de mercancías en el nivel internacional sin importar el papel que desempeñen. Según Hortal (2008), la figura de operador económico autorizado es común en las legislaciones y las reglamentaciones aduaneras; para estas organizaciones se establecen algunos beneficios tales como ahorro de tiempo y de costos, lo que se traduce en una serie de ventajas producto de las inversiones que las empresas hacen para el mejoramiento de la seguridad en la cadena de suministro. Ibáñez y Castillo (2011) aseguran que BASC contribuye al logro de la certificación de una empresa como operador económico autorizado, dado que compromete a toda la organización para cumplir con las reglas establecidas por el programa de seguridad de la Organización Mundial de Aduanas.

El sistema BASC utiliza como estrategia la colaboración entre todos los socios vinculados al comercio internacional; en este sentido Worl Basc Organization (2010) sustenta que: 
La cooperación se fundamenta principalmente en un intercambio permanente de experiencias, información y capacitación, lo cual ha permitido a las partes incrementar sus conocimientos y perfeccionar sus prácticas en un esfuerzo por mantener las compañías libres de cualquier actividad ilícita y a la vez facilitar los procesos aduaneros de las mismas.

Se puede concluir que BASC constituye una serie de estrategias de colaboración con el fin de que todas aquellas empresas que lo implementen garanticen a sus clientes y a todas las personas y organizaciones interesadas que sus procesos se desarrollan bajo estándares de seguridad aceptados internacionalmente. No obstante los beneficios mencionados, el BASC implica una reestructuración de los procesos y una serie de cambios en el nivel organizacional. El propósito de este trabajo es analizar y comprender los impactos positivos en lo operacional y financiero como resultado de la implementación de este sistema de gestión en las empresas que se han certificado con este estándar.

\section{Indicadores de productividad}

Berechet y San Miguel (2006) señalan que los indicadores de productividad permiten fijar una relación entre la cantidad de bienes y servicios producidos y la cantidad de recursos utilizados, transformándose en un factor crucial para la determinación del eficiente uso de los recursos. Otra manera de medición de la productividad es la planteada por el Banco Nacional de Comercio Exterior de México (1991), la cual se expresa como:

[...] un cambio cualitativo para hacer más y mejor las cosas, utilizar con racionalidad los recursos, participar más activamente en la innovación y los avances tecnológicos y conseguir la mayor concurrencia de la población en la actividad económica y en sus frutos.

Por tanto, la productividad se constituye en un elemento clave para la creación de riquezas dentro de una empresa, lo que permite la realización de inversiones en mejores recursos productivos (autofinanciamiento) como nuevas tecnologías; esta situación se traduce en una ventaja competitiva e incremento de los sueldos, lo que acrecentará el volumen de la demanda agregada, que resulta en el mejoramiento de la economía (Miranda y Toirac, 2010). Por otro lado, la medición de la productividad es un procedimiento necesario para el desarrollo y la proyección de las actividades económicas de cualquier organización; dicha medición se lleva a cabo 
mediante la aplicación de indicadores que relacionan diversas variables asociadas con las diferentes actividades y variables de transformación.

Dentro de una organización los indicadores de productividad se pueden medir con respecto a un factor de producción determinado, motivo por el cual existe la posibilidad de que se presenten una amplia gama de indicadores referentes a diferentes áreas, aunque los más importantes, según Miranda y Toirac (2010), son los relacionados con la productividad del trabajo, la productividad del uso de los materiales y la productividad del capital.

\section{Evaluación del impacto sobre la productividad mediante el análisis discriminante}

El análisis discriminante es una técnica de cálculo multivariable que permite identificar las variables o características que diferencian a un grupo de otro. Asimismo, sugiere el número de variables por tener en cuenta para poder clasificar los elementos dentro de su respectivo grupo de manera óptima; la variable dependiente en este tipo de análisis se toma como la pertenencia a determinado grupo, mientras que las variables independientes son las características que se supone diferencian a los elementos de cada grupo.

El estudio desarrollado por Suárez (2000) comprueba la efectividad de este análisis para la clasificación del nivel de rentabilidad de las empresas debido a la facilidad que éste establece para el manejo de múltiples variables. Vivanco et al. (2010) aplican este método para determinar los niveles de competitividad de varias empresas de acuerdo con ciertas variables específicas, lo que permite comprobar la utilidad que tiene el análisis discriminante para la clasificación de empresas que presentan variables similares, pero que es necesario diferenciarlas. Los autores anteriores dan cuenta de la efectividad de esta metodología para establecer la pertenencia o no de una empresa a un grupo que posee ciertas variables que determinan la competitividad, rentabilidad y productividad de dicho grupo.

Ahora, como resultado de esta revisión bibliográfica, se estructura y propone una metodología única para la aplicación del análisis discriminante en la evaluación de la incidencia de la certificación BASC en los indicadores de productividad de las empresas de Cartagena en los años 2008 y 2010. De esta manera se determina si existen diferencias significativas y mejoras en los indicadores de productividad seleccionados para estos años. 


\section{Metodología}

Este trabajo utiliza un análisis cualitativo, descriptivo propositivo y cuantitativo, soportado en un análisis discriminante multivariado (ADM), para contrastar si en dos periodos distintos se puede evidenciar la incidencia de la certificación BASC en la productividad financiera del sector analizado; para ello se estudiaron los rubros requeridos de las empresas de la ciudad de Cartagena (Vuran, 2009). Para la realización de esta investigación se utilizaron los indicadores presentados en el cuadro 1; del mismo modo se muestran las ecuaciones (1), (2), (3), (4), (5) y (6) en donde se específica como calcular los indicadores de productividad.

\section{Cuadro 1 \\ Indicadores de productividad ${ }^{1}$}

\begin{tabular}{|c|c|}
\hline Indicador & Ecuación \\
\hline IP1 & $\frac{\text { (Utilidad Bruta) }}{\text { (Valor agregado (ventas-pagos a proveedores+inventarios)) }} \mathrm{X} 100$ \\
\hline IP2 & $\frac{\text { (Utilidad Operacional) }}{(\text { Valor agregado (ventas-pagos a proveedores+inventarios)) }} \mathrm{X} 100$ \\
\hline IP3 & $\frac{\text { (Utilidad Neta) }}{\text { (Valor agregado (ventas-pagos a proveedores+inventarios)) }} \mathrm{X} 100$ \\
\hline IP4 & $\frac{\text { (Valor agregado (ventas-pagos a proveedores+inventarios) }}{\text { (Capital Operativo (activos corrientes y fijo)) }} \mathrm{X} 100$ \\
\hline IP5 & $\frac{\text { (Utilidad Operacional) }}{\text { (Capital Operativo (activos corrientes y fijo)) }} \times 100$ \\
\hline IP6 & $\frac{\text { (Utilidad Neta) }}{(\text { Capital Operativo (activos corrientes y fijo )) }}$ X100 \\
\hline
\end{tabular}

Asimismo, como criterio de selección de variables que mejor discriminan en la evaluación de la productividad como resultado de la certificación BASC en los indicadores seleccionados de las empresas de la ciudad de Cartagena se utilizó la distancia $\mathrm{D}^{2}$ de Mahalanobis, pues con su aplicación se determina la similitud o diferencia entre dos variables teniendo en cuenta la correlación entre cada una de ellas (Escobedo y Salas, 2008), lo que permite saber cuáles son las variables o indicadores con mayor variación.

\footnotetext{
${ }^{1}$ El valor agregado es el valor creado durante la prestación del servicio o la realización del producto. La utilidad bruta es la utilidad propia de los servicios que se prestan o los productos que se elaboran. La utilidad operacional es el resultado de tomar los ingresos operacionales y restarle los costos y gastos operacionales de los servicios prestados o productos elaborados. La utilidad neta resulta después de restar y sumar, de la utilidad operacional, los gastos e ingresos no operacionales, respectivamente, los impuestos y la reserva legal.
} 
Como cualquier otra técnica estadística la aplicación de la misma ha de ir precedida de una comprobación de los supuestos asumidos por el modelo. El análisis discriminante se apoya en los siguientes supuestos:

- Normalidad multivariante.

- Igualdad de matrices de varianza-covarianza.

- Linealidad.

- Ausencia de multicolinealidad y singularidad.

\section{Población}

La población de esta investigación está conformada por 23 empresas de la ciudad de Cartagena certificadas por la Alianza Empresarial para el Comercio Seguro o Business Anti-Smuggling Coalition (BASC) que presentaron sus estados financieros entre el año 2008 y 2010 en la superintendencia de sociedades.

\section{Fuentes y datos}

Se tomaron como fuentes los estados financieros de 2008 y 2010 de la Superintendencia de Sociedades (2011), información registrada en BASC, Colombia, y en la Cámara de Comercio de las empresas seleccionadas; de dichos estados se tomaron los diferentes rubros financieros para calcular los indicadores de productividad de las organizaciones de la ciudad de Cartagena, Colombia, labor que tomó seis meses de trabajo.

\section{Análisis}

Para analizar las variables asociadas a los indicadores seleccionados, se utilizó el análisis discriminante por medio del software SPSS statistics 19, aplicación con la cual se establecieron las funciones discriminantes y se estudiaron los diferentes estadísticos; esta técnica permitió la estimación en un marco único y analizar si los indicadores financieros evaluados en el mismo contexto presentan diferencias significativas en los dos periodos seleccionados. Después se verificó el cumplimiento de los supuestos requeridos para aplicar el análisis discriminante y de esta forma calcular y establecer la función objetivo con el fin de determinar que indicadores discriminaban mejor para evaluar la productividad como resultado de la certificación BASC en las empresas de la ciudad de Cartagena. También se calculó la 
precisión del modelo para predecir el comportamiento futuro de los indicadores en el sector. Por último, con los estadísticos, específicamente las medias de los indicadores para el sector, se analizó y evaluó el comportamiento y evolución de los diferentes indicadores de productividad finacieros en los dos periodos (2008 y 2010).

\section{Resultados}

\section{Verificación de los supuestos}

En el cuadro 2 se presentan los resultados de normalidad para los datos de los periodos 2008 y 2010, respectivamente, asociados a la prueba de Shapiro \& Wilk que requiere que los estadísticos estén por encima de 0.8 . Las pruebas de normalidad para las variables por separado de los dos periodos respectivos dan como resultado que algunas no se comportan como variables normales. Sin embargo, Lachenbruch (1975) ha demostrado que el análisis discriminante no es particularmente sensible a las violaciones de menor importancia de la hipótesis de normalidad. Tabachnick y Fidell (2001) hacen algunas precisiones acerca de la robustez de esta técnica en relación con el tamaño de las muestras, por lo que sugieren un tamaño de muestra mayor a 20, de grupos similares, para que el modelo sea robusto ante la violación del supuesto de multinormalidad. Para el caso de esta investigación se utilizaron 23 , por lo que se cumple con la condición requerida.

\section{Cuadro 2}

Prueba de Shapiro \& Wilk para la comprobación de la normalidad de los indicadores de productividad del año 2008 y 2010, respectivamente

\begin{tabular}{c|r|r|r|r|r|r}
\hline \multirow{2}{*}{ Indicador } & \multicolumn{3}{|c|}{ Shapiro-Wilk 2008 } & \multicolumn{3}{c}{ Shapiro-Wilk 2010 } \\
\cline { 2 - 7 } & Estadístico & Muestras & \multicolumn{1}{c|}{ Sig. } & Estadístico & Muestras & \multicolumn{1}{c}{ Sig. } \\
\hline IP1 & 0.717 & 23 & 0 & 0.644 & 23 & 0 \\
\hline IP2 & 0.811 & 23 & 0.001 & 0.779 & 23 & 0 \\
\hline IP3 & 0.893 & 23 & 0.018 & 0.973 & 23 & 0.757 \\
\hline IP4 & 0.869 & 23 & 0.006 & 0.871 & 23 & 0.007 \\
\hline IP5 & 0.928 & 23 & 0.1 & 0.95 & 23 & 0.294 \\
\hline IP6 & 0.954 & 23 & 0.351 & 0.955 & 23 & 0.371 \\
\hline
\end{tabular}


Homogeneidad de matrices de varianza-covarianza

El supuesto de igualdad de matrices de varianza-covarianza para los años 2008 y 2010 se comprobó con la prueba de Box, como se muestra en el cuadro 3.

\section{Cuadro 3}

\section{Resultados de la prueba de BOX}

\begin{tabular}{c|c|r}
\hline \multicolumn{2}{|c|}{ M de Box } & 52.059 \\
\hline \multirow{4}{*}{ F } & Aprox. & 2.114 \\
\cline { 2 - 3 } & gl1 & 21 \\
\cline { 2 - 3 } & gl2 & 7120.605 \\
\cline { 2 - 3 } & Sig. & 0.002 \\
\hline
\end{tabular}

Los resultados obtenidos del estadístico de contraste $\mathrm{M}=52.059$ y un valor de $\mathrm{F}=2.114$ con una probabilidad asociada $\mathrm{p}=0.002$ impide aceptar la hipótesis nula de igualdad de covarianzas de los grupos de discriminación; es decir, la capacidad explicativa de separación de los grupos es buena.

\section{Linealidad y multicolinealidad y singularidad}

Los supuestos de linealidad y multicolinealidad y singularidad no serán revisados dado que para la construcción del modelo de análisis discriminante se utilizó el método paso a paso, teniendo en cuenta el criterio de tolerancia para seleccionar las variables que son incluidas. De este modo, aquellas variables que presentan una correlación múltiple elevada con las restantes variables arrojarán una baja tolerancia y no serían consideradas de cara a la construcción de la función discriminante (Rodríguez y Moreno, 2011).

\section{Selección de las variables que mejor discriminan}

Para determinar que variables discriminan independientemente entre los grupos de 2008 y 2010 se estima la distancia D² de Mahalanobis y el Lambda Wilks para cada una de ellos, su correspondiente razón F y nivel de significancia para rechazar la hipótesis nula que las observaciones provienen de la misma población. Se consideró un nivel de significación de 0.05 para los estadísticos F para entrar y F para eliminar. Para ello se utilizaron los indicadores presentados en el cuadro 1. Cons- 
tituyendo las funciones discriminantes, el resultado final del modelo se muestra en el cuadro 4 y en las ecuaciones (7) y (8).

Con base en los resultados se procedió a calcular las funciones discriminantes para pronosticar a futuro los diferentes indicadores de productividad seleccionados en las empresas de la ciudad de Cartagena (ver ecuaciones (7) y (8)).

\section{Cuadro 4}

\section{Coeficientes de la función de clasificación}

\begin{tabular}{c|c|c}
\hline \multirow{2}{*}{ Indicador } & \multicolumn{2}{|c}{ Año } \\
\cline { 2 - 3 } & $\mathbf{2 0 0 8}$ & $\mathbf{2 0 1 0}$ \\
\hline IP1 & 9.914 & 10.208 \\
\hline IP2 & 22.433 & 18.308 \\
\hline IP3 & -12.723 & -16.925 \\
\hline IP4 & 4.767 & 4.359 \\
\hline IP5 & -33.264 & -29 \\
\hline IP6 & 7.947 & 9.27 \\
\hline (Constante) & -4.53 & -4.198 \\
\hline \multicolumn{2}{|c}{}
\end{tabular}

$\mathbf{Z}_{1}=\mathrm{IP} 1 *(9.914)+\mathrm{IP} 2 *(22.433)+\mathrm{IP} 3 *(-12.723)+\mathrm{IP} 4 *(4.767)+\mathrm{IP} 5 *(-33.264)$

$+\operatorname{IP6}^{*}(7.947)-(4.530)$

$\mathrm{Z}_{2}=\mathrm{IP} 1 *(10.208)+\mathrm{IP} 2 *(18.308)+\mathrm{IP} 3 *(-16.925)+\mathrm{IP} 4 *(4.359)+\mathrm{IP} 5 *(-29.000)$ + IP6*(9.270) - (4.198)

De este análisis se puede inferir que las dos poblaciones estudiadas presentan un error tipo I de $39.1 \%$, un error tipo II de $56.5 \%$, para una efectividad de clasificación total de $52.2 \%$, como se muestra en el cuadro 5.

\section{Cuadro 5}

\section{Resultados de la clasificación}

\begin{tabular}{l|c|c|c|c|c}
\hline \multirow{2}{*}{} & & \multicolumn{2}{|c}{$\begin{array}{c}\text { Grupo de pertenencia } \\
\text { pronosticado }\end{array}$} & \multirow{2}{*}{ Total } \\
\cline { 3 - 5 } & & Año & $\mathbf{2 0 0 8}$ & $\mathbf{2 0 1 0}$ & \\
\hline \multirow{4}{*}{ Original } & \multirow{2}{*}{ Recuento } & 2008 & 14 & 9 & 23 \\
\cline { 3 - 6 } & \multirow{2}{*}{$\%$} & 2010 & 13 & 10 & 23 \\
\cline { 3 - 6 } & & 2008 & 60.9 & 39.1 & 100 \\
\cline { 3 - 6 } & & 2010 & 56.5 & 43.5 & 100 \\
\hline
\end{tabular}


Evaluación de la productividad en las empresas de la ciudad de Cartagena

Cuando se revisan los estadísticos (media) de los indicadores seleccionados en el cuadro 6 de las empresas de la ciudad de Cartagena - como son Utilidad bruta/ Valor agregado (IP1), Utilidad operacional/Valor agregado (IP2), Utilidad neta/Valor agregado (IP3), Valor agregado/Capital operativo (IP4), Utilidad operacional/ Capital operativo (IP5) y Utilidad neta/Capital operativo (IP6) - se observa que las medias no mejoran en el transcurso del 2008 y 2010.

\section{Cuadro 6}

Medias de los indicadores de productividad

\begin{tabular}{c|c|c}
\hline \multirow{2}{*}{ Indicador } & \multicolumn{2}{|c}{ Año } \\
\cline { 2 - 3 } & $\mathbf{2 0 0 8}$ & $\mathbf{2 0 1 0}$ \\
\hline IP1 & .2659 & .2552 \\
\hline IP2 & .0440 & -.0022 \\
\hline IP3 & .0491 & .0052 \\
\hline IP4 & 1.4348 & 1.3165 \\
\hline IP5 & .0800 & .0539 \\
\hline IP6 & .0625 & .0385 \\
\hline
\end{tabular}

\section{Discusión}

$\mathrm{Al}$ analizar las funciones discriminantes generadas en el cuadro 4 se puede concluir que en el análisis de la evaluación de la productividad los indicadores IP1, IP5 e IP6 discriminan bien mejorando de un periodo a otro; es decir, presentan diferencias significativas estos tres indicadores para la población estudiada. Sin embargo, para el caso de los demás indicadores se puede observar que no se evidencia el mismo comportamiento en el transcurso de los años 2008 y 2010. En lo que respecta a las medias de los indicadores seleccionados se puede concluir que ninguno de ellos mejoró en el transcurso de los años analizados como resultado de la certificación BASC.

A pesar de que en esta investigación no se evidencia la mejora en los indicadores de productividad seleccionados como resultado de la certificación Business Anti-Smuggling Coalition (BASC), otros estudios similares, como los desarrollados por Fontalvo et al. (2011a) y Fontalvo et al. (2011b), han demostrado la incidencia de la certificación de otros estándares operacionales en el mejoramiento de indicadores de sectores empresariales. 
Otros autores como Avendaño y Varela (2010) también han demostrado que la adopción de estándares afecta de manera positiva la competitividad y productividad de la empresa o el sector que los implementa porque éstos representan la estandarización de los procesos y la producción de productos sanos; estos mismos autores utilizaron el análisis discriminante para medir el impacto de la adopción de estándares por parte de las empresas, lo cual les permitió concluir que tanto la competitividad como la productividad están influidas positivamente por la adopción de estándares.

Como resultado de este trabajo de investigación se ofrece a la comunidad empresarial y científica una metodología para evaluar la productividad o cualquier otro indicador o indicadores como resultado de la implementación de estándares asociados con las estructuras operativas de las organizaciones utilizando análisis discriminante.

\section{Conclusión}

En el trabajo de investigación desarrollado, en el que se aplicó el análisis discriminante, se utilizaron 23 empresas de la ciudad de Cartagena; el modelo presentó una baja efectividad en la clasificación de las dos poblaciones estudiadas. En la muestra original la precisión del modelo en el 2008 es $39.1 \%$ y en el 2010 es del $56.5 \%$; el promedio total de clasificación es del $52.2 \%$ de las empresas, lo que demuestra una confiabilidad buena para aquellos indicadores que presentaron diferencias significativas o discriminaron bien en los periodos estudiados como fueron IP1, IP5 e IP6.

De los resultados y la discusión de esta investigación se puede concluir que a pesar de que existen diferencias significativas en algunos indicadores, no existe evidencia estadística de la incidencia de los procesos de certificación BASC en el mejoramiento de la productividad financiera para los periodos estudiados en este sector empresarial. Sin embargo, el impacto de la certificación BASC en la productividad puede cambiar en otros sectores empresariales.

Este trabajo constituye un referente para desarrollar otras investigaciones que evalúen no sólo variables e indicadores asociados con la productividad, sino otras variables internas y externas en diferentes sectores que permitan medir eficiencia, eficacia, productividad y competitividad. De igual forma, la investigación permitió establecer una función objetivo para las empresas evaluadas en la ciudad de Carta- 
gena, con lo que se puede estudiar y analizar que indicadores discriminan mejor y se pueden así tomar acciones teniendo en cuenta el cálculo y estudio de los indicadores que presentaron una buena discriminación.

Se recomienda para futuros estudios incrementar el número de indicadores financieros e incorporar indicadores de competitividad; asimismo, se invita a analizar el comportamiento de otros sectores empresariales.

\section{Referencias}

Avendaño, B. y R. Varela (2010). La adopción de estándares en el sector hortícola de baja california. Estudios fronterizos (11): 171-202.

Banco Nacional de Comercio Exterior de México (1991). La productividad y la capacitación, pilares de la modernización. Revista de Comercio Exterior 41: 20-32.

BASC Colombia (2010). Seguridad y protección al comercio internacional. Disponible en http://www.basccolombia.org.

Berechet, C. y F. San Miguel (2006). Innovación y productividad en la economía de Navarra. Posicionamiento frente a la regiones europeas más avanzadas. Centro para la Competitividad de Navarra. Industria Gráfica S.A. (4): 14-25.

Blanchard, D. (2006). Supply Chain \& Logistics: Protecting The Supply Chain. Industry Week (2): 43-46.

Chacón, G. (2007). La contabilidad de costos, los sistemas de control de gestión y la rentabilidad empresarial. Actualidad Contable FACES (15): 29-45.

Correa, A. y R. Gómez (2010). Seguridad en la cadena de suministro basado en la norma ISO 28001 para el sector y sus derivados como estrategia para su competitividad. Boletin de Ciencias de la Tierra (28): 39-50.

Díaz, A. (2008). Las nuevas medidas de seguridad y sus efectos en las exportaciones agroalimentarias. Colección de Documentos CEPAL (8): 1-27. 
Escobedo, M. y J. P. Ch. Salas (2008). Mahalanobis y las aplicaciones de su distancia estadística. Cultura, Ciencia y Tecnología 5 (27): 13-21.

Fariñas, J. (2011). Comercio Internacional, Empresa y Competitividad. Cuadernos Económicos de ICE (82): 5-10.

Fontalvo, T., A. Mendoza, A. y J. Morelos (2011a). Evaluación del impacto de los sistemas de gestión de la calidad en la liquidez y rentabilidad de las empresas de la Zona Industrial de Mamonal (Cartagena, Colombia). Revista Católica del Norte (1): 1-28.

-, J. Morelos y E. de la Hoz (2011b). Aplicación del análisis discriminante para evaluar el mejoramiento de los indicadores financieros en las empresas del sector extracción de petróleo crudo y gas natural en Colombia. Revista Soluciones de Postgrado EIA (1): 1-16.

Hortal, R. (2008). La figura del operador económico autorizado. Estrategia Financiera (253): 54-58.

Ibáñez, N. y R. Castillo (2011). El BASC como plataforma del operador económico autorizado (OEA). E-Boletin BASC Venezuela (23): 1-4.

Ibarra, V. (2007). Hacia un comercio internacional con desarrollo sustentable. Desafíos (16): 11-28.

Ibarra, Y. (2008). Fronteras seguras y facilitación del comercio: Análisis de economía institucional. Gestión Política y Pública (17): 3-33.

Lachenbruch, P. (1975). Discriminant Analysis. Nueva York: Macmillan Pub Co.

Manuj, I. y J. Mentzer (2008). Global Supply Chain Risk Management Strategies. International Jornal of Physical Distribution and Logistic Management (abstract) (8): 192-223.

Maruchek, A., N. Greis, C. Mena y L. Cai (2011). Product safety and security in the global supply chain: issues, challenges and research opportunities. Journal of Operation Management (29): 707-720. 
Miranda, J. y L. Toirac (2010). Indicadores de productividad para la industria dominicana. Redalyc (12): 235-290.

Osorio, A. (2010). Impacto de las certificaciones ISO 9000 y BASC en los despachos de exportación definitiva de las agencias de aduana peruanas 20042008. Revista de Ciencias Empresariales de la Universidad de San Martin de Porres (1): 25-38.

Pfohl, H., H. Kölher y T. David (2010). State of the art in Supply Chain Management Risk research: Empirical and conceptual findings and a roadmap for the implementation in practice. Logistic Research (2): 33-44.

Pinzón, P., J. Sánchez, M. Vélez y D. Álvarez (2011). Sistemas de control para la gestión de canales de exportación independientes: un análisis exploratorio sobre su diseño y su uso. Revista de Contabilidad (14): 115-146.

Rodríguez, J. y A. Moreno (2011). Fragilidad financiera de las firmas en Colombia, 2000-2006. Un análisis discriminante de un modelo Minskano. Universidad Nacional de Colombia (14): 14 -36.

Samiee, S. (2007). Global Marketing Efectiveness via Alliances and Electronic Commerce in Businnes-to-Businees Market. Industrial Marketing Managemen (37): 3-8.

Sarathy, R. (2006). Security and the Global Supply Chain. Transportation Journal 45: $28-51$.

Suárez, J. (2000). Los parámetros característicos de las empresas manufactureras de alta rentabilidad: una aplicación del análisis discriminante. Revista Española de Financiación y Contabilidad (29): 443-481.

Superintendencia de Sociedades. Disponible en http://sirem.supersociedades.gov. $\mathrm{co} / \mathrm{SIREM} /$.

Tabachnick, B. y L. Fidell (2001). Using Multivariate Statistics, 4a. ed. Boston: Pearson. 
Valdevira, O., E. Díaz y R. Sans (2009). Impacto y situación de los operadores de carga terrestre de mercancías en relación a la normativa actual en materia de gestión de la seguridad en la cadena de suministro, como paso para obtener el estatus de operador seguro. Estudios de Construcción y Transporte (111): 65-73.

Vivanco, M., F. Martínez e I. Taddei (2010). Análisis de competitividad de cuatro sistema-producto estatales de tilapia en México. Estudios Sociales (18): 165-207.

Vuran, B. (2009). Prediction of business failure: A comparison of discriminant and logistic regression analyses. Istanbul University journal of the school of business administration (38): 47-65.

Worl Basc Organization (2010). Business for Secure Commerce: What Is BASC? Disponible en http://www.wbasco.org.

Young, R. y P. Esqueda (2005). Vulnerabilidades de la cadena de suministros: Consideraciones para el caso de América Latina. Revista Latinoamericana de Administración (34): 63-78. 